Bull. Korean Math. Soc. 48 (2011), No. 5, pp. 1041-1046

http://dx.doi.org/10.4134/BKMS.2011.48.5.1041

\title{
A NEW KIND OF THE LAW OF THE ITERATED LOGARITHM FOR PRODUCT OF A CERTAIN PARTIAL SUMS
}

\author{
QING-PEI ZANG
}

\begin{abstract}
Let $\left\{X, X_{i} ; i \geq 1\right\}$ be a sequence of independent and identically distributed positive random variables. Denote $S_{n}=\sum_{i=1}^{n} X_{i}$ and $S_{n}^{(k)}=S_{n}-X_{k}$ for $n \geq 1,1 \leq k \leq n$. Under the assumption of the finiteness of the second moments, we derive a type of the law of the iterated logarithm for $S_{n}^{(k)}$ and the limit point set for its certain normalization.
\end{abstract}

\section{Introduction and the main result}

Let $\left\{X_{n} ; n \geq 1\right\}$ be a sequence of random variables and define the partial sum $S_{n}=\sum_{i=1}^{n} X_{i}$ and $S_{n}^{(k)}=S_{n}-X_{k}$ for $n \geq 1,1 \leq k \leq n$. In the past decades, the asymptotic behaviors of the products of various random variables have been widely studied. Arnold and Villaseñor [1] considered sums of records and obtained the following form of the central limit theorem (CLT) for independent and identically distributed (i.i.d.) exponential random variables with the mean equal to one,

$$
\frac{\sum_{k=1}^{n} \log S_{k}-n \log (n)+n}{\sqrt{2 n}} \stackrel{d}{\rightarrow} \mathcal{N} \text { as } n \rightarrow \infty .
$$

Here and in the sequel, $\mathcal{N}$ is a standard normal random variable, and $\stackrel{d}{\rightarrow}$ stands for convergence in distribution. In particular, Rempala and Wesolowski [13] removed the condition that the distribution is exponential and showed the asymptotic behavior of products of partial sums holds for any sequence of i.i.d. positive random variables. Namely, they proved the following theorem.

Theorem A. Let $\left\{X_{n} ; n \geq 1\right\}$ be a sequence of i.i.d. positive square integrable random variables with $E X_{1}=\mu, \operatorname{Var} X_{1}=\sigma^{2}>0$ and the coefficient of variation

Received March 26, 2010.

2010 Mathematics Subject Classification. 60F15.

Key words and phrases. law of the iterated logarithm, product of partial sums, strong law of large numbers. 
$\gamma=\frac{\sigma}{\mu}$. Then we have

$$
\left(\frac{\prod_{k=1}^{n} S_{k}}{n ! \mu^{n}}\right)^{\frac{1}{\gamma \sqrt{n}}} \stackrel{d}{\rightarrow} e^{\sqrt{2} \mathcal{N}} \text { as } n \rightarrow \infty
$$

This convergence may be applied in mathematical statistics in a rather routine way, for example, to construct the distribution free tests for the coefficient of variation $\gamma$. Recently, the above result was swiftly extended by Qi [12] and $\mathrm{Lu}$ and $\mathrm{Qi}[10]$ to a general limit theorem covering the case when the underlying distribution is integrable and belongs to the domain of attraction of a stable law with index from the interval $[1,2]$. Furthermore, this type of convergence was extended in different directions by many authors who considered dependent sequences, self-normalized sequences, almost sure limit theorem, law of the iterated logarithm, weak invariance principle and references therein. In this note, by the method of Qi [12], we establish a new type of the law of the iterated logarithm for $S_{n}^{(k)}$ for $n \geq 1,1 \leq k \leq n$.

Theorem 1.1. Let $\left\{X, X_{i} ; i \geq 1\right\}$ be a sequence of independent and identically distributed positive random variables. Denote $\mu=E(X)>0$, the coefficient of variation $\gamma=\sigma / \mu$, where $\sigma^{2}=\operatorname{Var}(X)$, and $S_{n}=\sum_{i=1}^{n} X_{i}$ and $S_{n}^{(k)}=S_{n}-X_{k}$ for $n \geq 1,1 \leq k \leq n$. Then

$$
\begin{array}{r}
\limsup _{n \rightarrow \infty}\left(\frac{\prod_{j=1}^{n} S_{n}^{(j)}}{(n-1)^{n} \mu^{n}}\right)^{\frac{1}{\gamma \sqrt{2 n \log \log n}}}=e, \text { a.s. } \\
\liminf _{n \rightarrow \infty}\left(\frac{\prod_{j=1}^{n} S_{n}^{(j)}}{(n-1)^{n} \mu^{n}}\right)^{\frac{1}{\gamma \sqrt{2 n \log \log n}}}=e^{-1}, \text { a.s. }
\end{array}
$$

and the limit point set is

$$
P\left(\mathcal{C}\left\{\left(\frac{\prod_{j=1}^{n} S_{n}^{(j)}}{(n-1)^{n} \mu^{n}}\right)^{\frac{1}{\gamma \sqrt{2 n \log \log n}}}\right\}=\left[e^{-1}, e\right]\right)=1 .
$$

\section{Proof of the main result}

Let $C$ denote positive constants whose values may vary from place to place. The notation of $a_{n}=o\left(b_{n}\right)$ means $\frac{a_{n}}{b_{n}} \rightarrow 0$ as $n \rightarrow \infty$, and $a_{n}=O\left(b_{n}\right)$ means $\frac{a_{n}}{b_{n}} \rightarrow C$ as $n \rightarrow \infty$, also let $\log y=\log (y \vee e), \log \log y=\log \log \left(y \vee e^{e}\right)$ for all $y>0$.

Proof of Theorem 1.1. By the expansion of the logarithm, we have

$$
\lim _{n \rightarrow \infty}\left(\prod_{j=1}^{n} \frac{\left(\log \frac{j+1}{j}\right)}{\frac{1}{j}}\right)^{\frac{1}{\gamma \sqrt{2 n \log \log n}}}=1 .
$$


In order to complete the proof of our main result, it suffices to prove

$$
\begin{array}{r}
\limsup _{n \rightarrow \infty}\left(\prod_{j=1}^{n} \frac{j\left(\log \frac{j+1}{j}\right) S_{n}^{(j)}}{(n-1) \mu}\right)^{\frac{1}{\gamma \sqrt{2 n \log \log n}}}=e, \text { a.s., } \\
\liminf _{n \rightarrow \infty}\left(\prod_{j=1}^{n} \frac{j\left(\log \frac{j+1}{j}\right) S_{n}^{(j)}}{(n-1) \mu}\right)^{\frac{1}{\gamma \sqrt{2 n \log \log n}}}=e^{-1} \text {, a.s.. }
\end{array}
$$

First, by virtue of the strong law of large numbers for $S_{n}^{(k)}, k \geq 1$, we have

$$
\frac{S_{n}^{(k)}-(n-1) \mu}{n-1} \rightarrow 0, \text { a.s. as } n \rightarrow \infty .
$$

Then, as $j \rightarrow \infty$, we have

$$
\begin{aligned}
\varepsilon_{j} & :=\frac{j\left(\log \frac{j+1}{j}\right) S_{n}^{(j)}}{(n-1) \mu}-1 \\
& =j\left(\log \frac{j+1}{j}\right) \frac{S_{n}^{(j)}-(n-1) \mu}{(n-1) \mu}+j\left(\log \frac{j+1}{j}\right)-1 \rightarrow 0, \text { a.s.. }
\end{aligned}
$$

For $4 / 3<r<2$, by Marcinkiewics-Zygmund strong law of large numbers, we have

$$
\left(\frac{S_{n}^{(j)}-(n-1) \mu}{(n-1) \mu}\right)^{2}=o\left(n^{\frac{2}{r}-2}\right), \text { a.s.. }
$$

Thus

$$
\sum_{j=1}^{n}\left(j \log \frac{j+1}{j}\right)^{2}\left(\frac{S_{n}^{(j)}-(n-1) \mu}{(n-1) \mu}\right)^{2}=o\left(n^{\frac{2}{r}-1}\right), \text { a.s. }
$$

which coupled with $\sum_{j=1}^{n}\left(j \log \frac{j+1}{j}-1\right)^{2}=O(1)$. Then

$$
\begin{aligned}
\sum_{j=1}^{n} \varepsilon_{j}^{2} & \leq C \sum_{j=1}^{n}\left(j \log \frac{j+1}{j}\right)^{2}\left(\frac{S_{n}^{(j)}-(n-1) \mu}{(n-1) \mu}\right)^{2}+C \sum_{j=1}^{n}\left(j \log \frac{j+1}{j}-1\right)^{2} \\
& =o\left(n^{\frac{2}{r}-1}\right), \text { a.s.. }
\end{aligned}
$$

In view of $\frac{2}{r}-1<\frac{1}{2}$, we have

$$
\frac{\sum_{j=1}^{n} \varepsilon_{j}^{2}}{\sqrt{n \log \log n}} \rightarrow 0, \text { a.s. as } n \rightarrow \infty \text {. }
$$

By (2.4) and the expansion of logarithm, we have

$$
\frac{j\left(\log \frac{j+1}{j}\right) S_{n}^{(j)}}{(n-1) \mu}=1+\varepsilon_{j}=e^{\varepsilon_{j}+O\left(\varepsilon_{j}^{2}\right)}, \text { a.s. },
$$


combining with (2.6), we have

$$
\begin{aligned}
\left(\prod_{j=1}^{n} \frac{j\left(\log \frac{j+1}{j}\right) S_{n}^{(j)}}{(n-1) \mu}\right)^{\frac{1}{\gamma \sqrt{2 n \log \log n}}} & =e^{\frac{\sum_{j=1}^{n} \varepsilon_{j}}{\gamma \sqrt{2 n \log \log n}}+O\left(\frac{\sum_{j=1}^{n} \varepsilon_{j}^{2}}{\gamma \sqrt{2 n \log \log n}}\right)} \\
& =e^{\frac{\sum_{j=1}^{n} \varepsilon_{j}}{\gamma \sqrt{2 n \log \log n}}+o(1)}, \text { a.s.. }
\end{aligned}
$$

Thus, it suffices to prove

$$
\begin{aligned}
& \limsup _{n \rightarrow \infty} \frac{\sum_{j=1}^{n} \varepsilon_{j}}{\gamma \sqrt{2 n \log \log n}}=1, \text { a.s., } \\
& \liminf _{n \rightarrow \infty} \frac{\sum_{j=1}^{n} \varepsilon_{j}}{\gamma \sqrt{2 n \log \log n}}=-1, \text { a.s. }
\end{aligned}
$$

and the limit point set is

$$
P\left(\mathcal{C}\left\{\frac{\sum_{j=1}^{n} \varepsilon_{j}}{\gamma \sqrt{2 n \log \log n}}\right\}=[-1,1]\right)=1 .
$$

Indeed, we have

$$
\begin{aligned}
\frac{\sum_{j=1}^{n} \varepsilon_{j}}{\gamma \sqrt{2 n \log \log n}}= & \frac{1}{\sigma \sqrt{2 n \log \log n}} \sum_{j=1}^{n}\left(j \log \frac{j+1}{j}\right)\left(\frac{S_{n}^{(j)}-(n-1) \mu}{n-1}\right) \\
& +\frac{\mu}{\sigma \sqrt{2 n \log \log n}} \sum_{j=1}^{n}\left(j \log \frac{j+1}{j}-1\right) \\
= & \frac{1}{\sigma \sqrt{2 n \log \log n}} \sum_{j=1}^{n}\left(j \log \frac{j+1}{j}\right)\left(\frac{S_{n}^{(j)}-(n-1) \mu}{n-1}\right) \\
& +O\left(\frac{\log n}{2 \sigma \sqrt{n \log \log n}}\right) \\
= & \frac{S_{n}-n \mu}{\sigma \sqrt{2 n \log \log n}} \frac{\sum_{j=1}^{n}\left(j \log \frac{j+1}{j}\right)}{n-1}-\frac{\sum_{j=1}^{n}\left(j \log \frac{j+1}{j}\right)\left(X_{j}-\mu\right)}{\sigma(n-1) \sqrt{2 n \log \log n}} \\
& +o(1) \\
= & : I_{n}+I I_{n}+o(1),
\end{aligned}
$$

for $I_{n}$, together with $\lim _{n \rightarrow \infty} \frac{\sum_{j=1}^{n}\left(j \log \frac{j+1}{j}\right)}{n-1}=1$, and the well known HartmanWintner law of the iterated logarithm theorem, we have

$$
\begin{aligned}
& \limsup _{n \rightarrow \infty} I_{n}=1, \text { a.s., } \\
& \liminf _{n \rightarrow \infty} I_{n}=-1, \text { a.s., }
\end{aligned}
$$

and every point of the closed interval $[-1,1]$ (in the sense of almost sure convergence) for the sequence $\left\{I_{n}\right\}$. Now we are in a position to deal with $I I_{n}$. 
From Markov's inequality, for any $\varepsilon>0$, we have

$$
\begin{aligned}
\sum_{n \geq 2}^{\infty} P\left(I I_{n}>\varepsilon\right) & \leq \sum_{n \geq 2}^{\infty} \frac{\operatorname{Var}\left(\frac{\sum_{j=1}^{n}\left(j \log \frac{j+1}{j}\right)\left(X_{j}-\mu\right)}{\sigma(n-1) \sqrt{2 n \log \log n}}\right)}{\varepsilon^{2}} \\
& \leq C \sum_{n \geq 2}^{\infty} \frac{\sum_{j=1}^{n}\left(j \log \frac{j+1}{j}\right)^{2}}{(n-1)^{2} n \log \log n} \\
& \leq C \sum_{n \geq 2}^{\infty} \frac{1}{(n-1)^{2} \log \log n}<\infty
\end{aligned}
$$

then, by Borel-Cantelli lemma, one has

$$
\lim _{n \rightarrow \infty} I I_{n}=0, \text { a.s.. }
$$

Thus, we complete the proof of $(2.7),(2.8),(2.9)$. Now, the proof of our main result is derived.

Acknowledgement. The authors would like to thank professor Zhengyan Lin of Zhejiang University for his help.

\section{References}

[1] B. C. Arnold and J. A. Villaseñor, The asymptotic distributions of sums of records, Extremes 1 (1999), no. 3, 351-363.

[2] P. Y. Chen, On the law of iterated logarithm for products of sums, Acta Math. Sci. Ser. A Chin. Ed. 28 (2008), no. 1, 66-72.

[3] K. A. Fu and W. Huang, A weak invariance principle for self-normalized products of sums of mixing sequences, Appl. Math. J. Chinese Univ. Ser. B 23 (2008), no. 2, 183189.

[4] K. Gonchigdanzan, An almost sure limit theorem for the product of partial sums with stable distribution, Statist. Probab. Lett. 78 (2008), no. 18, 3170-3175.

[5] K. Gonchigdanzan and K. M. Kosiński, On the functional limits for partial sums under stable law, Statist. Probab. Lett. 79 (2009), no. 17, 1818-1822.

[6] K. Gonchigdanzan and G. Rempala, A note on the almost sure limit theorem for the product of partial sums, Appl. Math. Lett. 19 (2006), no. 2, 191-196.

[7] K. M. Kosiński, On the functional limits for sums of a function of partial sums, Statist. Probab. Lett. 79 (2009), no. 13, 1522-1527.

[8] Y. X. Li and J. F. Wang, Asymptotic distribution for products of sums under dependence, Metrika 66 (2007), no. 1, 75-87.

[9] W. D. Liu and Z. Y. Lin, Asymptotics for self-normalized random products of sums for mixing sequences, Stoch. Anal. Appl. 25 (2007), no. 4, 739-762.

[10] X. W. Lu and Y. C. Qi, A note on asymptotic distribution of products of sums, Statist. Probab. Lett. 68 (2004), no. 4, 407-413.

[11] P. Matula and I. Stepien, Weak convergence of products of sums of independent and non-identically distributed random variables, J. Math. Anal. Appl. 353 (2009), no. 1, 49-54.

[12] Y. C. Qi, Limit distributions for products of sums, Statist. Probab. Lett. 62 (2003), no. $1,93-100$.

[13] G. Rempala and J. Wesolowski, Asymptotics for products of sums and v-statistics, Electron. Comm. Probab. 7 (2002), 47-54. 
[14] L. X. Zhang and W. Huang, A note on the invariance principle of the product of sums of random variables, Electron. Comm. Probab. 12 (2007), 51-56.

School of Mathematical SCiEnCE

Huaiyin Normal University

Huaian 223300, P. R. China

E-mail address: zqphunhu@yahoo.com.cn 